\title{
A Neural-Wavelet Technique for Damage Identification in the ASCE Benchmark Structure Using Phase II Experimental Data
}

\author{
Mahmoud M. Reda Taha \\ Department of Civil Engineering, The University of New Mexico, Albuquerque, NM 87131, USA \\ Correspondence should be addressed to Mahmoud M. Reda Taha, mrtaha@unm.edu \\ Received 15 December 2009; Revised 18 March 2010; Accepted 25 May 2010 \\ Academic Editor: Yiqing Qing Ni
}

Copyright ( $) 2010$ Mahmoud M. Reda Taha. This is an open access article distributed under the Creative Commons Attribution License, which permits unrestricted use, distribution, and reproduction in any medium, provided the original work is properly cited.

\begin{abstract}
Damage pattern recognition research represents one of the most challenging tasks in structural health monitoring (SHM). The vagueness in defining damage and the significant overlap between damage states contribute to the challenges associated with proper damage classification. Uncertainties in the damage features and how they propagate during the damage detection process also contribute to uncertainties in SHM. This paper introduces an integrated method for damage feature extraction and damage recognition. We describe a robust damage detection method that is based on using artificial neural network (ANN) to compute the wavelet energy of acceleration signals acquired from the structure. We suggest using the wavelet energy as a damage feature to classify damage states in structures. A case study is presented that shows the ability of the proposed method to detect and pattern damage using the American Society of Civil Engineers (ASCEs) benchmark structure. It is suggested that an optimal ANN architecture can detect damage occurrence with good accuracy and can provide damage quantification with reasonable accuracy to varying levels of damage.
\end{abstract}

\section{Introduction}

With the aging of infrastructure worldwide and the increasing availability of cost efficient sensing equipment, the necessity to implement damage identification and classification systems on civil structures has become imperative. Structural health monitoring (SHM) is the nonintrusive collection and analysis of data from structures for damage detection and diagnosis. The intention of SHM is to characterize the structure's performance and to help maintain the structural performance over its years of service. SHM also helps reduce operation costs through early damage detection. Successful SHM techniques have been applied to other engineering disciplines where the mass of the structure is small compared with civil structures. Vibration-based SHM assumes that the structural dynamic response will depart from its normal pattern when damage occurs in the structure. Thus, damage detection is contingent upon successfully extracting sensitive damage feature(s), patterning such feature(s) and realizing changes in these patterns as damage develops.
Over the past two decades numerous research methods with the objective of extracting sensitive damage feature(s) have been suggested and tested on several structures [15]. Tools for damage detection using structural dynamics analysis such as modal update, Fourier transform and wavelets have been examined [6]. Some of those pertaining to the ASCE benchmark structure, described in Section 2, are reviewed below in Table 1 .

More recently, a few researchers have focused on the use of artificial neural networks (ANN) for damage pattern recognition. ANN consists of a group of interconnected processing units called neurons. Each neuron performs a simple computational process and has a transfer function associated with the layer that operates at the node level. ANN has the capability to learn from example datasets by changing the numerical biases and weights of the network [40]. For feed forward ANN that is considered here, the neurons are organized into layers where the first layer contains one neuron per input and the last layer contains one neuron per output; intermediate layers may contain any number of layers. While Tsou and Shen [41] used differences between 
TABLE 1: Summary of damage detection methods for the ASCE benchmark structure.

\begin{tabular}{|c|c|c|}
\hline Reference & Author(s) & Damage detection method \\
\hline \multirow{2}{*}{$\begin{array}{l}\text { Reference } \\
\text { document-No } \\
\text { data }\end{array}$} & Johnson et al. $[7,8]$ & Detailed Description of Phase I-Simulated \\
\hline & Dyke et al. [9] & Detailed Description of Phase II-Experimental \\
\hline \multirow{15}{*}{$\begin{array}{l}\text { Phase I: Simulated } \\
\text { data }\end{array}$} & Dyke et al. [10] & Loss of stiffness of members byoptimizing modal parameters \\
\hline & Hera et al. [11] & Spikes in Level 1 details of wavelet decomposed signals \\
\hline & Yang et al. [12] & Spectral analysis to identify stiffness parameters \\
\hline & Hera and Hou [13] & Spikes in Level 1 details of wavelet decomposed signals \\
\hline & Sun and Chang [14] & Covariance of response using wavelet packets \\
\hline & Lam et al. [15] & Loss of stiffness using modal update and identification \\
\hline & Yuen et al. [16] & $\begin{array}{l}\text { Loss of stiffness of members using modal parameter extraction and Bayesian } \\
\text { modal updating }\end{array}$ \\
\hline & $\begin{array}{l}\text { Lus et al. and Caicedo } \\
\quad \text { et al. }[17,18]\end{array}$ & $\begin{array}{l}\text { State space model, eigensystem realization algorithm and optimization using } \\
\text { modal parameters }\end{array}$ \\
\hline & $\begin{array}{l}\text { Bernal and Gunes } \\
\qquad[19]\end{array}$ & Extraction of a matrix proportional to structure flexibility \\
\hline & Lin et al. [20] & $\begin{array}{l}\text { Time-frequency features obtained using Hilbert-Huang transform of the } \\
\text { intrinsic mode functions }\end{array}$ \\
\hline & Chase et al. [21] & Recursive least square to identify changes in stiffness matrix \\
\hline & $\mathrm{Wu}$ and $\mathrm{Li}[22]$ & Eingen-sensitive FE for damage detection in ambient vibration \\
\hline & Yang and Huang [23] & A recursive nonlinear estimation method is used \\
\hline & $\begin{array}{l}\text { Mizuno and Fujino } \\
{[24]}\end{array}$ & Haar wavelet decomposition, quantization, and dissimilarity \\
\hline & Zhou et al. [25] & Residual values from subspace-modal identification \\
\hline \multirow{3}{*}{$\begin{array}{l}\text { Phase II: Simulated } \\
\text { data }\end{array}$} & Hou and Hera [26] & $\begin{array}{l}\text { Spikes in Level } 1 \text { details of wavelet decomposed signals using Daubechies and } \\
\text { Meyer wavelets }\end{array}$ \\
\hline & $\begin{array}{c}\text { Barroso and } \\
\text { Rodriguez [27] }\end{array}$ & Comparison of healthy to damage curvature in the mode shapes \\
\hline & Casciati $[28]$ & Discrepancy between healthy and damaged states using sum of squared errors \\
\hline \multirow{3}{*}{$\begin{array}{l}\text { Phase II: Simulated } \\
\text { and experimental } \\
\text { data }\end{array}$} & Hera and Hou [29] & Modal parameters determined using continuous wavelet transform \\
\hline & Dincal and Raich [30] & Minimization of error term between FRF of experimental \& simulated data \\
\hline & Nair et al. [31] & $\begin{array}{l}\text { Structural stiffness change based on poles; pattern classification with } \\
\text { autoregressive coefficients }\end{array}$ \\
\hline \multirow{6}{*}{$\begin{array}{l}\text { Phase II: } \\
\text { experimental data } \\
\text { only }\end{array}$} & $\begin{array}{l}\text { Ching and Beck } \\
\qquad[32,33]\end{array}$ & $\begin{array}{l}\text { Expectation-Maximization algorithm used to find most probable stiffness } \\
\text { parameters-Config. } 2-9\end{array}$ \\
\hline & Giraldo et al. [34] & Loss of stiffness of members-Config 2-6 \\
\hline & Lynch [35] & Pole location using system identification, Config. $1-5$ \\
\hline & Liu et al. [36] & $\begin{array}{l}\text { Time-frequency obtained using Hilbert-Huang transform of intrinsic } \\
\text { modes-Config. } 7 \text { \& } 8\end{array}$ \\
\hline & $\begin{array}{l}\text { McCuskey et al. [37] } \\
\text { and McCuskey [38] }\end{array}$ & Neural-wavelet module_-All Configurations \\
\hline & $\begin{array}{c}\text { Carden and } \\
\text { Brownjohn [39] }\end{array}$ & $\begin{array}{l}\text { Autoregressive moving average (ARMA) to build damage classifiers for } \\
\text { different damage configurations }\end{array}$ \\
\hline
\end{tabular}

healthy and damaged eigenvalues as training data for a neural network for damage detection of a spring-mass system, Sexton et al. [42] proved that optimizing neural networks using optimization can lead to better prediction capabilities in operation research modeling.
The application of such stiffness-based techniques to large civil structures has been challenging because of the insignificant effect of the relatively small changes in stiffness due to damage compared with the large mass of these structures. Elkordy et al. [43] trained a neural network 
using a finite element model for a large civil structure and compared the results to a physical model. The ability to train a neural network with finite element data is critical for evaluation of structures where sets of data representing healthy performance may not exist.

Several signal processing methods have been promoted for feature extraction such as Fourier transform, Wavelet transform and Wavelet Multi-Resolution Analysis (WMRA) $[6,44,45]$. These methods were combined with means of artificial intelligence (AI) such as ANN [46]. It has been noted by Lam et al. [47] and Yuen and Lam [48] that the influence of ANN architecture has been overlooked by many researchers using ANN for damage pattern recognition. The ANN architecture is crucial to the training of the network as well as getting good performance from the ANN. Lam et al. [47] suggested the use of a Bayesian method (conditional probability) to determine the optimal ANN architecture while using a Ritz vector and modal parameters, respectively, as damage features. A dual function ANN was used for the process of feature extraction and then to establish the needed damage classifier. The use of ANN for establishing nonlinear classifiers has also been suggested by other researchers [49]. A review of a number of combination of AI tools and signal processing techniques, particularly wavelets, for damage feature extraction for SHM, has recently been reported [45]. The use of wavelets and combining ANN and Wavelets for damage detection has also been recommended by investigators Yam et al. [50], Kim and Melhem [51], Diao et al. [52], Jiang and Adeli [53] and Jiang and Mahadevan [54, 55].

Much of the above noted research was focused on damage feature extraction rather than on damage pattern recognition. Sohn et al. [56] suggested classifying damage in structures using statistical pattern recognition methods. Lam et al. [47] discussed the possible use of Bayesian analysis to identify damage classes. Other techniques using fuzzy (nonprobabilistic) damage pattern recognition were reported to enable realizing other types of uncertainty, rather than random uncertainty in damage detection [57].

In this paper, we suggest using available damage observations to identify the optimal ANN structure (i.e., number of hidden layers and number of neurons in each hidden layer). An optimization process is suggested to identify the optimal ANN structure for successful damage pattern classification. Here we used acceleration data collected experimentally from Phase II of the ASCE benchmark structure to develop and test the proposed damage pattern recognition method. Our motivation was to demonstrate the possible use of an optimized neural-wavelet module to detect and quantify damage with reasonable accuracy in the ASCE benchmark structure. The proposed framework is extendable for damage detection and quantification in other structures.

\section{The ASCE Benchmark Structure: Background}

The American Society of Civil Engineers (ASCE) benchmark study was conducted by the International Association for Structural Control (IASC) ASCE Structural Health Monitoring Task Group as a resource for validating damage detection techniques. The ASCE Benchmark Group generated structural response data from a $2 \times 2$ bay, four story, rectangular steel test structure [9]. A schematic of the structure is shown in Figure 1(a).

Phase I of the ASCE benchmark study was generated by means of a finite element model considering varying levels of damage $[7,8]$. Phase II included two parts: Phase II-S where "S" denotes simulation data and Phase II-E where "E" denotes experimental data. The data in Phase II-S was generated using structural dynamics finite element software under MATLAB environment [7]. Phase II$S$ model included a 120 degrees of freedom (DOF) model and a twelve DOF data model $[7,8]$. Phase II-E included experimental data collected from the structural response of the ASCE benchmark rectangular steel structure tested at the University of British Columbia in August 2002 [9]. Table 1 presents a review of all previous research that examined the ASCE benchmark structure to date. It is obvious that the vast amount of this previous research used simulated data, not experimental data. Table 1 also provides a brief description of the damage detection techniques used by each researcher and the type of structural response considered for the cases where experimental data of the benchmark structure was used.

As presented in Table 1, many researchers had success in detecting damage in Phase I and Phase II-S of the benchmark structure, but similar success when using the same techniques to Phase II-E data have not been reported. For example, Nair et al. [31] showed promising results for detecting damage using the Phase II-S data from the benchmark problem with a pattern classification algorithm based on autoregressive analysis of acceleration signals in the time domain. However, Nair et al. [31] reported limited success in using Phase II-E data. Therefore, the research conducted on the experimental benchmark study is not complete or fully inclusive. A major limitation in most of previous work examining the benchmark structure was the focus on damage detection rather than damage quantification. As most researchers tried to validate their metrics for damage detection of the different scenario, no attempts were made to produce an overall damage quantification metric based on the damage feature elected for damage detection. Here, we try to provide a method that provides both damage detection and damage quantification and demonstrate its application to the ASCE benchmark structure.

The ASCE benchmark structure was built at approximately one-third scale and is located at the University of British Columbia [9]. A series of acceleration data was collected from the test structure using nine different levels of damage and three different types of excitation including a shaker using a sine sweep, random excitation and impulse testing [58]. Only the response data from the randomly excited structure was analyzed. Phase II-E data includes acceleration response recorded for Phase I structural configurations. These configurations are described in Table 2. It is important to note that such description in Table 2 does not provide a quantitative value of the level of damage. Therefore, judging a damage detection method becomes quite challenging. This is because of the fact that it is difficult to quantify how removal of one or more 


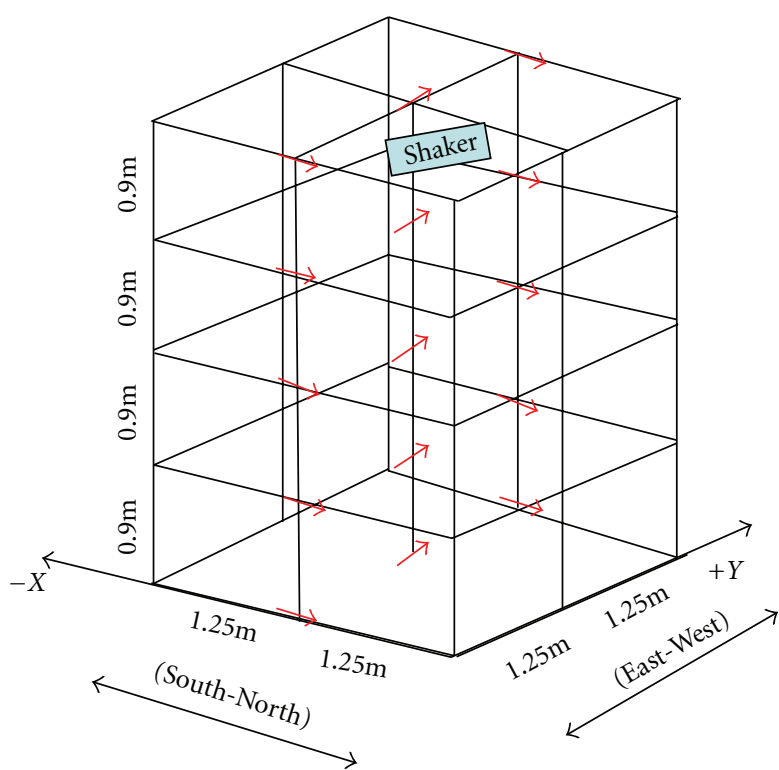

(a)

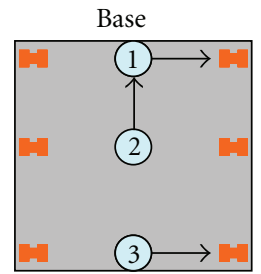

3rd floor

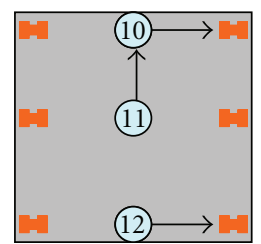

(12) $\longrightarrow$

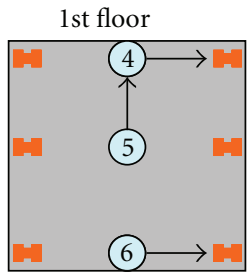

4th floor

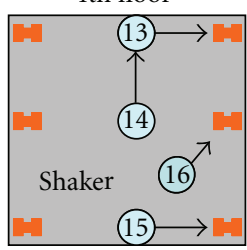

(b)
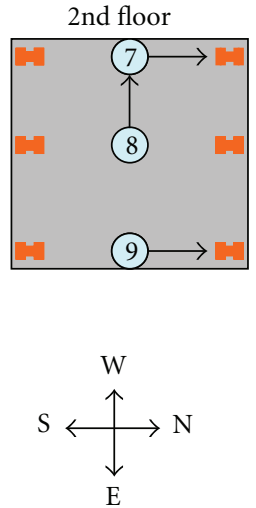

FIGURE 1: (a) 3D schematic of the ASCE benchmark structure. (b) Location of the accelerometers and shaker on the ASCE benchmark structure.

braces will affect structural response. While we propose a simple system to represent the level of damage based on the number of braces removed or joints loosened, we argue that further discussion from the SHM research community is needed to benchmark the levels of damage in the ASCE benchmark structure. Such benchmarking is essential for the experimental data's use by the research community when examining new damage detection methods.

Our proposed damage severity metric, denoted $\Gamma_{\text {ASCE }}$ describes damage in the ASCE benchmark structure, is defined and normalized to a scale between 0 and 100 for the benchmark configurations. The damage severity $\Gamma_{\mathrm{ASCE}}^{i}$ for the $i$ th damage configuration is computed as

$$
\Gamma_{\mathrm{ASCE}}^{i}=\frac{(1.0) n_{x}^{i}+(0.5) n_{y}^{i}+(0.25) B_{x}^{i}+(0.1) B_{y}^{i}}{\max \left((0.1) n_{x}+(0.5) n_{y}+(0.25) B_{x}+(0.1) B_{y}\right)} .
$$

The symbol $n_{x}$ is used to denote the number of braces removed in the North-South (strong axis) direction, while $n_{y}$ corresponds to the number of braces removed in the East-West (weak axis) direction. $B_{x}$ denotes the number of loosened bolted connections in the North-South (strong axis) direction while $B_{y}$ is the number of loosened bolts in the East-West (weak axis) direction. Weight factors of 1.0, $0.5,0.25$ and 0.1 are used to describe the significance of each action (e.g., removal of braces) on the level of damage in the structure. For instance, a weight factor of 1.0 is chosen for removal of braces in the strong axis (North-South) direction. The weight factor $(0.5)$ is used to describe the effect of removed bracing braces in the out-of-plane direction (EastWest) direction. The weight factor $(0.25)$ is also used to represent the relatively low effect of loosened bolts compared to the effect of removed bracingbraces. The factor $(0.1)$ is used to represent the effect of out-of-plane loosened bolts. The reduction of damage severity in the out-of-plane direction is attributed to the fact that most accelerometers were placed in the North-South direction and the accelerations of interest used in the analysis were in the North-South direction. Figures 1(a) and 1(b) shows a three-dimensional schematic of the ASCE benchmark structure and the location of the accelerometers and shaker on the structure. There are nine testing configurations tested in the experimental investigation of the ASCE benchmark structure [9]. There was an unspecified error in data reported for Configuration 5 according to ASCE Benchmark Group, therefore that dataset for Configuration 5 was not used in this study. The descriptive damage metric $\Gamma_{\text {ASCE }}$ was calculated for the eight damage configurations by means of (1). The damage metric values for these configurations are presented in Table 2. It became obvious that Configuration 1 can be classified as "healthy" $\left(\Gamma_{\mathrm{ASCE}}=0\right)$, Configurations $2-6$ can be classified as "partially damaged" with $\Gamma_{\text {ASCE }}$ ranging between 8.5 and 31.2 and Configurations 7-9 can be classified as "fully damaged" with $\Gamma_{\text {ASCE }}$ ranging between 85.1 and 100. It is important to emphasize that the proposed descriptive damage metric developed for validation of the proposed damage detection method is very specific to the ASCE benchmark structure and the testing configurations examined herein. The weight factors were specifically selected for quantifying the overall damage in the benchmark structure given the structural configurations and the sensor locations.

\section{Methods}

Here we suggest a computational method for feature extraction and damage recognition based on integrating ANN and WMRA. The proposed method is used for damage feature 
TABle 2: Damage cases and quantified damage metric $\left(\Gamma_{\mathrm{ASCE}}\right)$ based on experimental description of damage in ASCE benchmark structure.

\begin{tabular}{lcc}
\hline Structure Configuration & $\Gamma_{\mathrm{ASCE}}$ & Damage class \\
\hline $\begin{array}{l}\text { (1) Fully braced } \\
\text { (2) All braces removed from East face }\end{array}$ & 28.4 & Healthy \\
(3) Braces removed on all floors in SE & 21.3 & PD \\
corner & & \\
$\begin{array}{l}\text { (4) Braces removed 1 \& 4 floors, SE } \\
\text { corner }\end{array}$ & 8.5 & PD \\
$\begin{array}{l}\text { (5) Removed braces on E \& N faces (2nd } \\
\text { floor) }\end{array}$ & 31.2 & PD \\
$\begin{array}{l}\text { (6) Braces removed all floors and all faces } \\
\text { (7) Config. 7 plus loosened bolts N \& E }\end{array}$ & 105.1 & FD \\
face \\
(8) Config. 7 plus loosened bolts N \& E \\
face (1st \& 2nd floors)
\end{tabular}

extraction by realizing the changes in the energy of structural acceleration signals computed in the wavelet domain as a result of damage. The proposed method has been previously validated using simulated and experimental data on bridge structures $[59,60]$. Moreover, optimization methods are used to establish a classifier that can provide efficient damage pattern recognition. Design of the classifier is based on minimizing the error of classification by the damage detection method. The classification error is minimized through identifying the optimal architecture of the neural network, including the number of layers and number of neurons in each layer.

The development of the integrated damage pattern recognition method outlined in this paper includes the following steps: (1) data acquisition and signal processing, (2) damage feature extraction, (3) development of a damage classifier, (4) optimization of the neural network architecture using the classifier and (5) evaluation of the integrated damage recognition method (in Section 4).

3.1. Data Acquisition and Signal Processing. The acceleration signals, denoted $x_{i}(n)$ with subscript $i$ representing the sensor location, were processed using WMRA. WMRA was implemented using the discrete wavelet transform (DWT), specifically the daubechies (db4) mother wavelet [61]. Extensive study has been performed by the authors that showed the daubechies (db4) mother wavelet to be the most suitable wavelet to decompose acceleration signals [61]. Other wavelet functions proposed by other researchers [62, 63] were also examined. The energy of the approximation signal of the damage structures (as will be discussed below) showed more sensitivity to damage when the daubechies (db4) wavelet signal was used to decompose the original signal. Similar observation was reported on analysis of experimental data observed in monitoring of steel bridges [64].

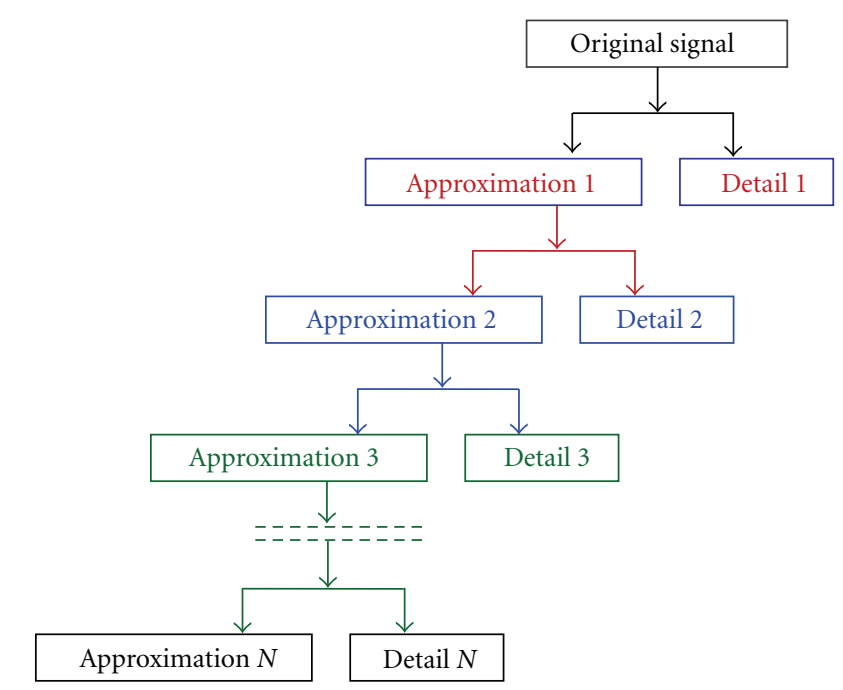

FIGURE 2: Schematic representation of wavelet multi-resolution analysis showing the decomposition of the original signal into $N$ levels of decomposition and details signals.

WMRA enables the decomposition of the acceleration signal in the time domain into component signals at different frequency levels named approximations and details. Scaling a wavelet simply means stretching or compressing it. The smaller the scale, the more the wavelet will be compressed while the larger the scale, the more the wavelet will be stretched. Therefore, low scales allow analyzing rapidly changing details (high frequency components) and high-scales allow analyzing slowly changing features (lowfrequency components).

In civil structures, it has been shown that most of the main frequency components are low-frequency components (ranging $5-30 \mathrm{~Hz})[6,31,64,65]$. Therefore, the lowfrequency components of the signal (the approximations) are very important parts of the signal. The "approximations signal" corresponds to the high-scale, low-frequency components of the signal. On the other hand, the high frequency contents carry the details of the signal. The "details signal" corresponds to the low scale high frequency part. WMRA decomposes the signal into various resolution levels as schematically shown in Figure 2. We suggest here using the third approximation signal in lieu of the original signal. This means that all high frequency components of the signal will be neglected. It is important to emphasize that these high frequency components might include useful information about the structure, but the proposed approach is based on the assumption that this information is not necessary for damage detection. The use of high frequency components might provide further insight on damage but will be accompanied with high computational expenses. The threshold at which signal decomposition is limited and the frequency components considered for damage detection shall be based on balancing the computational expenses versus the level of enhancement in damage detection accuracy [66].

Consider the discrete acceleration signal $x(n)$. With the wavelet scaling index $(j)$ and shifting index $(k)$ defined, the 
coefficients for the approximation signals $\left(a_{j, k}\right)$ and detail signals $\left(d_{j, k}\right)$ can be calculated as

$$
\begin{gathered}
a_{j, k}=2^{(-j / 2)} \sum_{n} x(n) \phi\left(2^{-j} n-k\right) \\
d_{j, k}=\sum_{n} x(n) \psi_{j, k}(n),
\end{gathered}
$$

where $\phi(n)$ is the scaling function [61]. The acceleration signals acquired from the ASCE benchmark structure were decomposed to three levels of decomposition. The third approximation signal was used to represent the structure's response.

3.2. Damage Feature Extraction. The proposed framework aims at establishing the complex relationship relating the structural dynamics (here accelerations) between different zones of the healthy structure. This general framework is shown schematically in Figure 3(a). The neural-wavelet module tries to build this relationship at the wavelet decomposed acceleration signals using artificial neural network. When damage occurs in the structure, the relationship between its zones is disturbed. The higher the severity of damage, the further the structure departs from the healthy relationship between its zones. Damage can be thus detected and classified.

We suggest a damage feature denoted $\lambda$ computed using WMRA and ANN. Acceleration signals recorded at accelerometers 5, 6, 9, 12 and 15 were decomposed using WMRA and the third approximation of the signals were then used as inputs for the ANN. The third approximation of the acceleration signal at accelerometer 13 was used as the desired output as shown in Figure 3(b). Figure 3(b) shows a schematic representation of the computation of the damage feature $\lambda$ in the ASCE benchmark structure.

The damage feature was obtained by comparing the monitored acceleration signal at sensor 13 and the signal predicted by ANN. The damage feature denoted $\lambda$ can thus be calculated as:

$$
\lambda=\sum_{k}\left|a_{J}(k)\right|^{2},
$$

where $a$ is the approximation, $J$ is the level of wavelet decomposition. $\lambda$ describes the energy of the signal representing the difference between the ANN predicted signal and the third approximation of the monitored acceleration signals and $k$ is the total number of discrete elements used to represent the signal. As ANN is trained to predict healthy performance, the difference between monitored and predicted signal represents the level of departure of the structural response at any time. This different signal is directly related to the level of damage in the structure. The lower the value of $\lambda$, the closer ANN's prediction is to the monitored signal, therefore representing a healthy structure. The higher the value of $\lambda$, the further ANN's prediction is from the monitored signal. This neural wavelet method has successfully identified damage in other structures, including prestressed concrete [60] and a structural steel model bridge [64].
Here, we only considered the structure's response data from accelerometers 5, 6, 9, 12, 13 and 15 shown on the benchmark structure plans described in Figure 3(b). These accelerometers were excited by an electro-dynamic shaker placed on the top floor of the structure. The choice of this group of accelerometers to form the damage detection module is based on their location in relation to the damaged areas (areas of bracing removal) of the ASCE benchmark structure configurations as described by the ASCE benchmark study report ASCE Benchmark Group [58] and Dyke et al. [9]. The accelerometers chosen as the inputs to the ANN were primarily positioned along the East face of the structure where damage was induced. The output accelerometer was positioned on the West face of the structure where no damage was induced.

ANNs use an iterative process to learn a pattern and generate a nonlinear mapping system between system inputs and output. Here, ANN is used to learn the complex healthy signal of the structure at sensor 13 by observing the signals at sensors 5, 6, 9, 12 and 15. The input layer of ANN in this study has five neurons corresponding to the five acceleration data inputs acquired by accelerometers (5, 6, 9, 12 and 15) and one neuron at the output layer acquired by accelerometer 13. Each neuron has a transfer function associated with the layer that operates at the node level. All layers use the log-sigmoid transfer function, with the exception of the output layer, which has a linear transfer function. The choice of these transfer functions was based on a parametric investigation conducted on the benchmark data [37]. The parametric investigation also showed the need for optimization of the ANN architecture to enable efficient damage classification.

3.3. Developing Damage Classifier. The neural network's ability to accurately mimic the healthy signal is dependent upon the architecture of ANN. In this study we target identifying the optimal architecture of ANN and defining the number of hidden layers and the number of neurons per layer such that damage detection is maximized. Design of ANN usually targets achieving a minimum training error which is considered a modeling tool criterion for acceptance of a neural network. Successful damage detection necessitates that the damage feature $(\lambda)$ is able to classify the level of damage in the structure. This means high $\lambda$ values correspond to the "highly damaged" benchmark Configurations 7 , 8 , and 9. Moreover, low $\lambda$ values correspond to the "healthy" Configuration 1 , and $\lambda$ values in between these extremes represent the "partially damaged" Configurations 2,3 , 4, and 6.

Successful development shall enable ANN to function as a damage classifier in addition to its role in damage feature extraction. This can be achieved by finding the optimal ANN architecture such that the maximum success rate of the damage classification is achieved. The process of successful classification rate maximization is performed here in the context of system optimization where the objective function that is used for defining the classifier is minimized. The optimization process can be described by defining 


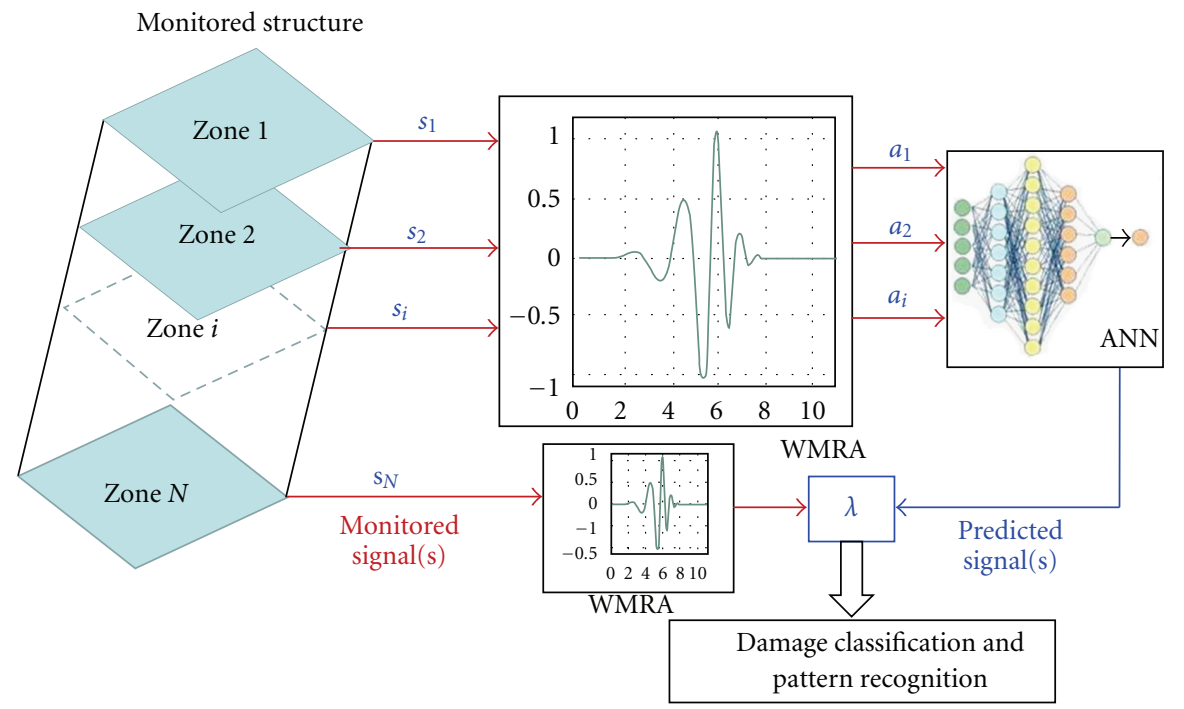

(a)
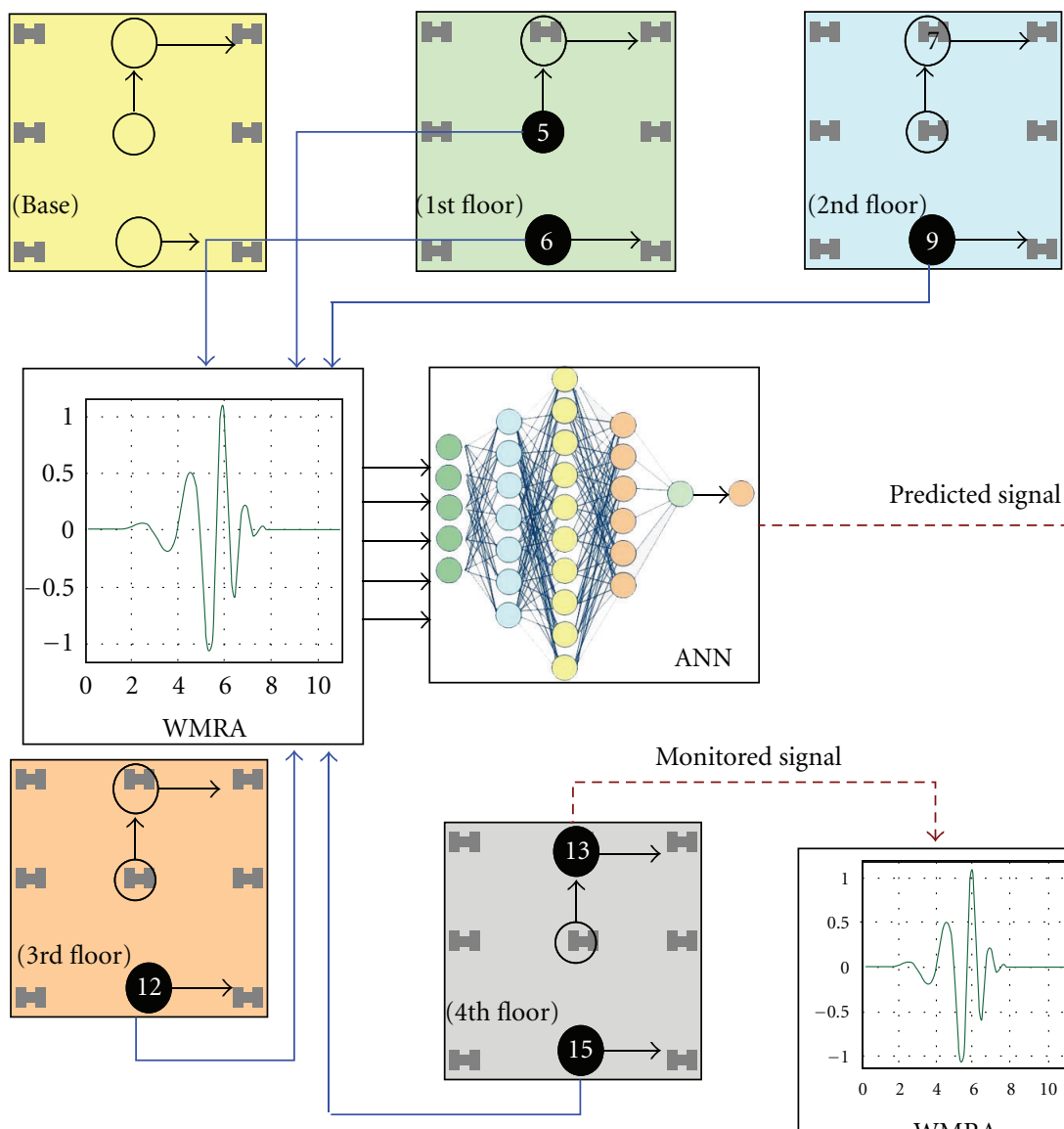

Monitored signal
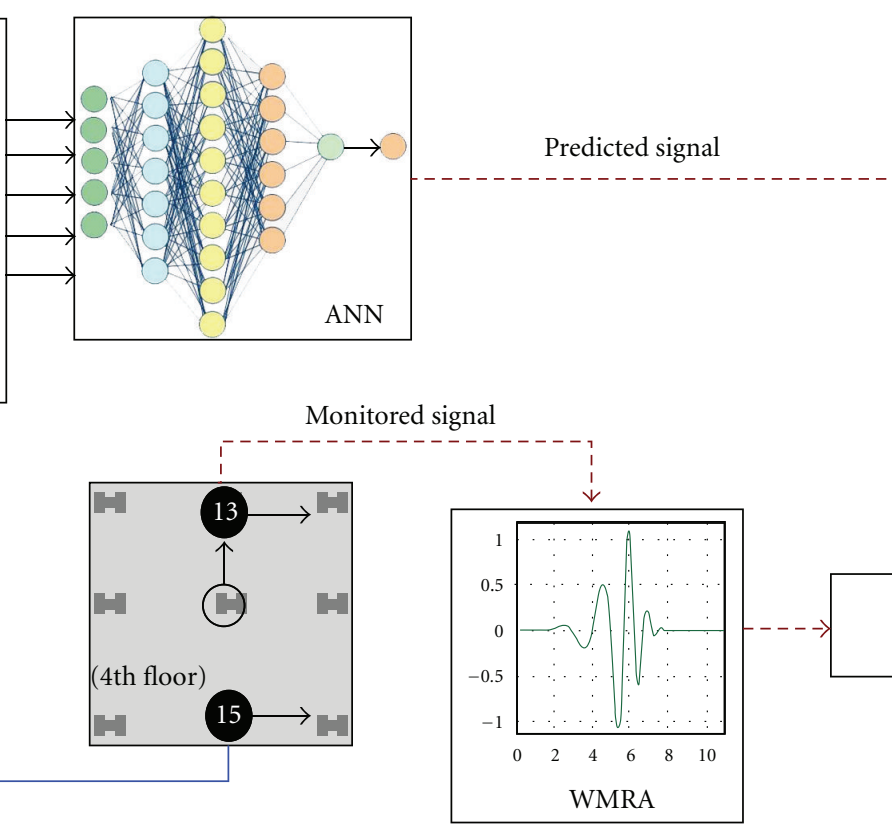

(b)

Figure 3: Schematic representation of the neural-wavelet damage detection method (a) general application of method to any structure divided to $N$ zones (b) specific method application for damage detection in the ASCE benchmark structure (damage feature $\lambda$ ). 


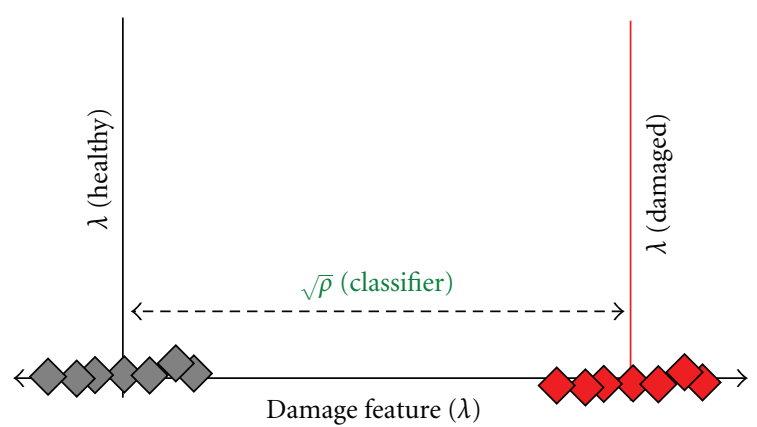

Figure 4: $\sqrt{\rho}$ represents the distance between mean value of $\lambda$ for healthy performance and the mean value of $\lambda$ for a damaged state.

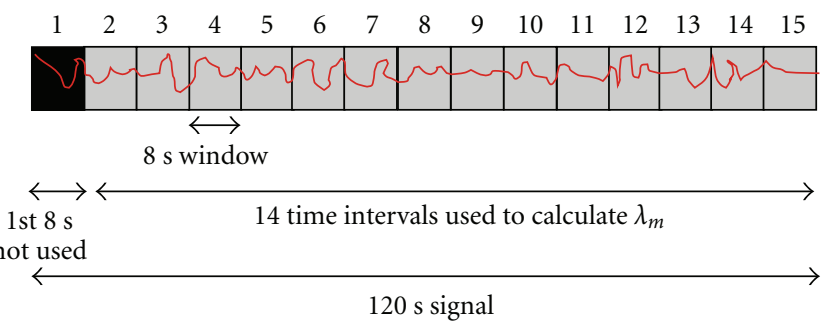

FIGURE 5: Schematic representation of the acceleration signals and the 15 windows in the signals.

the objective function, the design variables, the design parameters and the optimization constraints.

The objective function to be minimized is $-\rho$ with $\rho$ defined as:

$$
\rho=\sum_{i=1}^{K}\left(\lambda_{m}^{H}-\lambda_{m_{i}}^{D}\right)^{2}
$$

$\rho$ is the damage classifier, $\lambda_{m^{i}}^{H}$ is the mean value of the damage feature for a healthy case and $\lambda_{m^{i}}^{D}$ is the mean value of the damage feature for the damaged case. Where $i$ is a numerical counter, $i=1,2, \ldots, K . K$ represents the number of structural damage configurations considered when establishing the damage classifier. Defining $-\rho$ as the objective function ensures that the difference between the mean damage feature at the healthy performance and other damage states is maximized and thus the success rate of damage detection is maximized. A schematic representation of the function describing the damage classifier is shown in Figure 4.

The design variables describe the number of layers and the number of neurons in each layer of the ANN architecture. The design parameters include those parameters that affect the optimization process but are assumed constant during the optimization process. This includes the level of wavelet decomposition, $J$ (here $J=3$ ), the mother wavelet function (here $\mathrm{db} 4$ ) and the transfer function per ANN layer (here logsigmoid). Two optimization constraints were considered; the number of neurons per hidden layer constrained between 1 and 15 , and the number of hidden layers also constrained between 1 and 3. Both constraints were established to limit computation time. The optimization process was

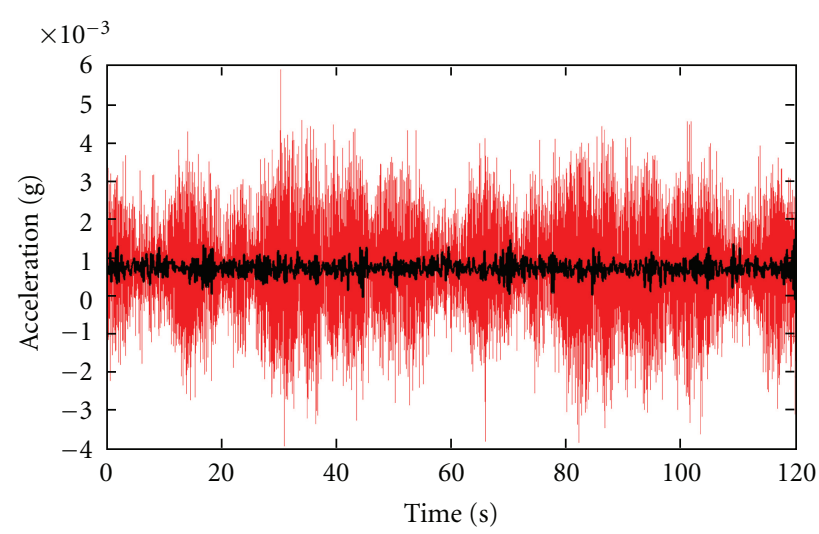

Raw acceleration data
Third approximation

FIGURE 6: Raw acceleration signal (red) observed at sensor 9 in the benchmark structure versus the third approximation of the acceleration signal (black) used for damage detection.

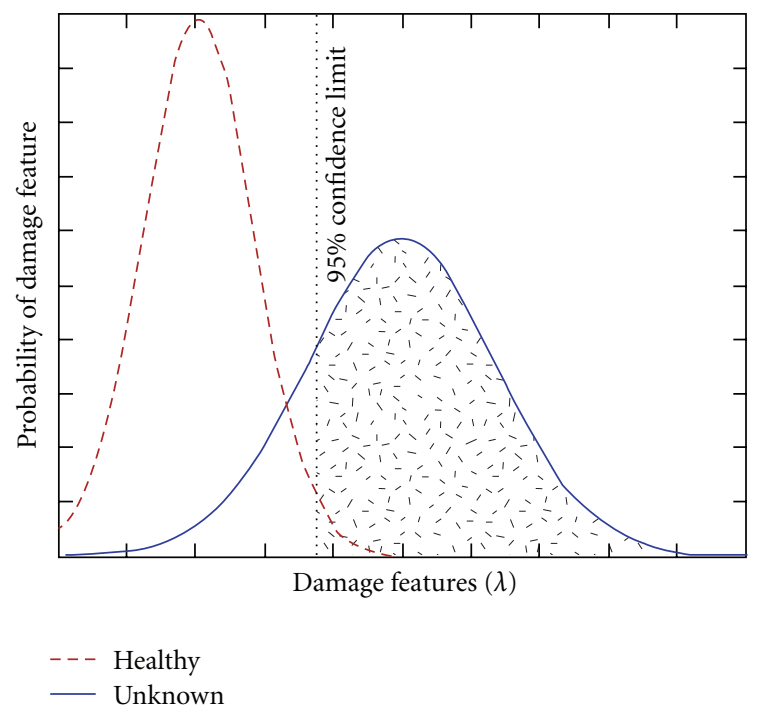

FIgURE 7: Schematic representation of probability of damage. Hatched area represents the cumulative probability of damage representing the neural-wavelet damage metric $\Gamma_{\mathrm{NW}}$.

performed using derivative-based (Newton gradient-decent) and derivative-free (genetic algorithm (GA)) optimization techniques [67, 68].

Considering the ASCE benchmark Phase II-E data, $\lambda_{m}^{H}$ represents the mean value of the damage feature for the healthy Configuration 1, while $\lambda_{m^{i}}^{D}$ represents the mean value of the damage feature of the ith damage configuration in the ASCE benchmark structure. Benchmark Configurations 1, 6, 7 and 8 were used for training of the damage classifier $\rho$, while Configurations 2, 3, 4 and 9 were reserved for testing the classifier after establishing the optimal ANN architecture. The mean value of the damage feature for the $i$ th damage configuration, $\lambda_{m^{i}}^{D}$, was computed as the mean of fourteen values of $\lambda$. Each $\lambda$ represents the energy of an 


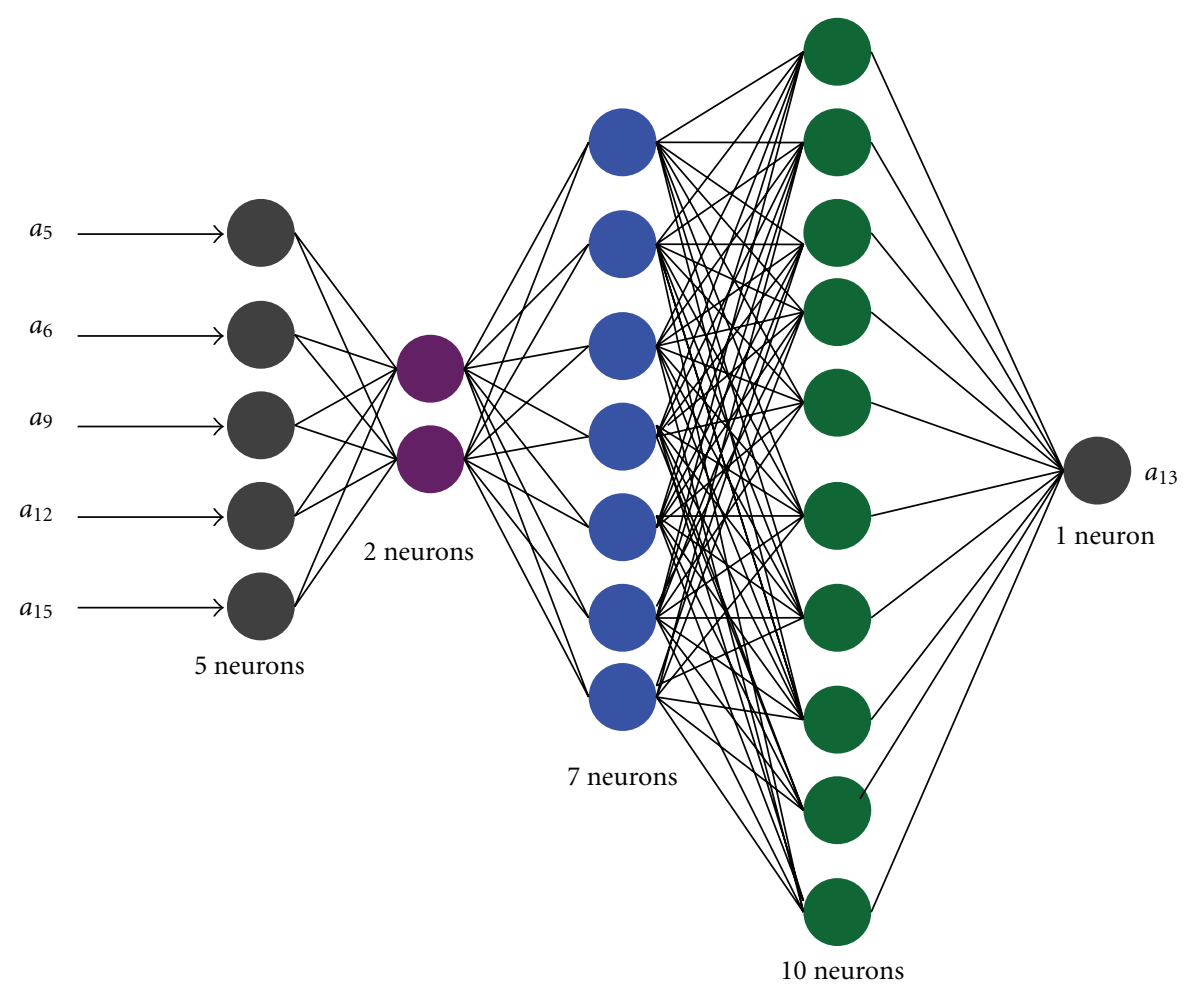

Figure 8: Optimal ANN for damage detection using the neural-wavelet method.

eight second window of the signal representing the difference signal between the third approximation of the measured signal and the ANN predicted signal. The energy of the difference signal is computed as

$$
\lambda=\frac{1}{T} \sum_{N=1}^{T} \sum_{k}\left|a_{d}(k)\right|^{2}
$$

$a_{d}$ is the difference signal between the third approximation of the measured signal and the ANN predicted signal, $k$ is the counter for the signal measurements within the 8 second window, $N$ represents the eight second time window and ranges from 1 to $T$, which is the total number of windows, equals 14. The division of the acceleration signal into windows is presented schematically in Figure 5. The original acceleration signal was 120 seconds long sampled at $500 \mathrm{~Hz}$ with 4000 data points in each window. The original signal was decomposed using WMRA. The original signal and the third approximation signal of a typical acceleration signal are shown in Figure 6. The third approximation signal was divided into fifteen windows each 8 seconds long. The first window was neglected for avoiding inaccurate observations at the start of data acquisition. The following 14 windows were used to compute the damage feature $\lambda$ as represented by (5). The WMRA decomposition was performed on the whole 120 second signal and not at the windows' level to avoid the well known edge effect of the wavelet analysis when short signals are analyzed.
3.4. Testing the Damage Classifier. The optimal ANN architecture and the damage classifier were tested using the testing data including Configurations 2, 3, 4 and 9. These data sets were not used in developing the damage classifier. To consider uncertainty in damage recognition, the probability of damage is used as the damage metric to represent the level of damage in the structure for each testing configuration. The damage metric denoted $\Gamma_{\mathrm{NW}}^{i}$ (denoting damage quantified using the neural-wavelet (NW) method) at the ith configuration of the benchmark structure can be evaluated using (6), where $\theta$ is the confidence limit established by considering a 95\% level of confidence from the healthy performance and $\lambda^{i}$ is the mean damage feature describing the $i$ th configuration of the benchmark structure

$$
\Gamma_{\mathrm{NW}}^{i}=P\left(\lambda^{i} \geq \theta\right),
$$

where $P$ represents the probability that the damage feature $\lambda$ will exceed the damage threshold $\theta$. The probability of damage is schematically represented in Figure 7 . The probability distribution function represents the damage feature at any instance. The probability of damage is calculated as the area under the probability density function of the damage state with for values higher than the confidence limit, $\theta$. Therefore, a probability density function for a damage case that does not overlap with the healthy probability density function would have a $100 \%$ probability of damage. The suggested method for calculating the probability of damage assumes the damage feature to be monotonically increasing as the level of damage in the structure advances. The damage 


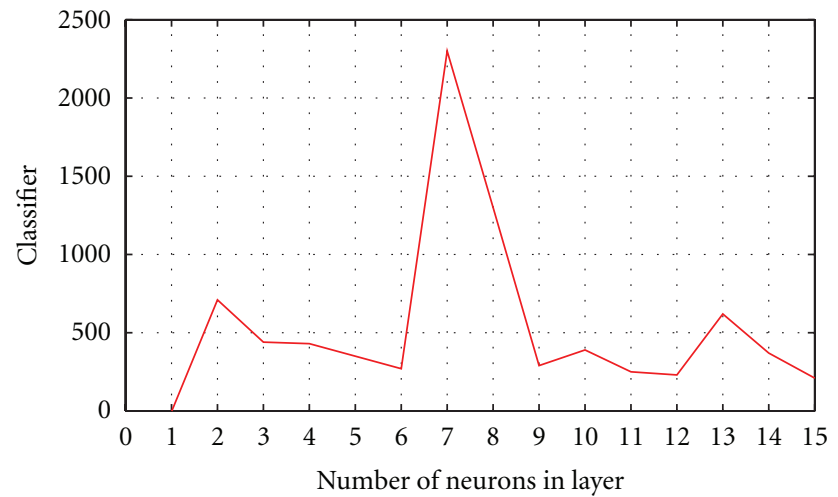

(a)

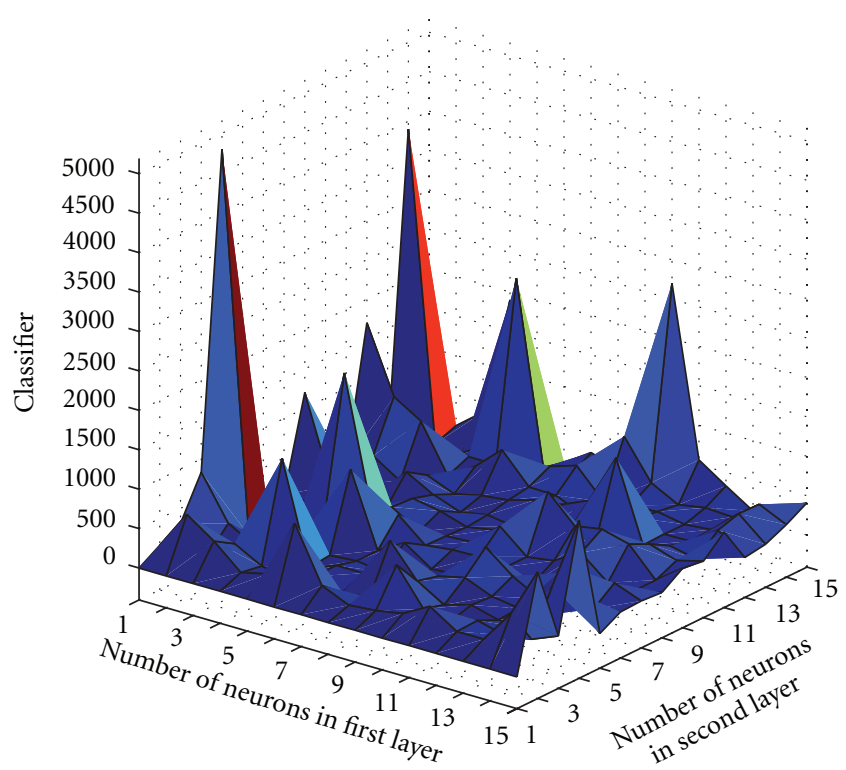

(b)

Figure 9: Number of neurons versus classifier objective function. (a) One hidden layer for classifier $(\rho)$ showing the peak classifier to occur at $n=7$ neurons. (b) Two hidden layers for classifier $(\rho)$ showing the peak classifier to occur at $n_{1}=1$ and $n_{2}=5$.

feature is also assumed to follow the normal (Gaussian) probability distribution which was confirmed by analyzing the healthy datasets.

\section{Results and Discussion}

The optimization process determined that the optimal ANN architecture includes three hidden layers consisting of 2, 7 and 10 neurons on the first, second and third hidden layers respectively. A schematic representation of the optimal network is shown in Figure 8. Figure 9 shows the change in the classifier objective function versus the number of neurons for classifier $\rho$ when the number of hidden layers is one (Figure 9(a)) and for two hidden layers in Figure 9(b). It is interesting to observe the large number of local minima that exist in both domains. The GA optimization technique was

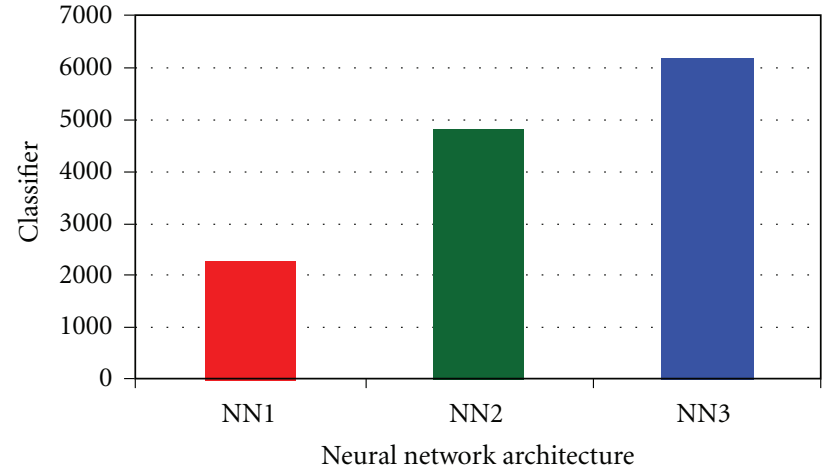

Figure 10: Comparison between three optimal neural networks using one hidden layer denoted NN1 ( $n_{1}=7$ neurons), two hidden layers denoted NN2 $\left(n_{1}=1\right.$ and $\left.n_{2}=5\right)$ and three hidden layers denoted NN3 $\left(n_{1}=2\right.$ and $n_{2}=7$ and $\left.n_{3}=10\right)$ showing the neural network with three hidden layers to have the best performance with the highest classifier $(-\rho)$.

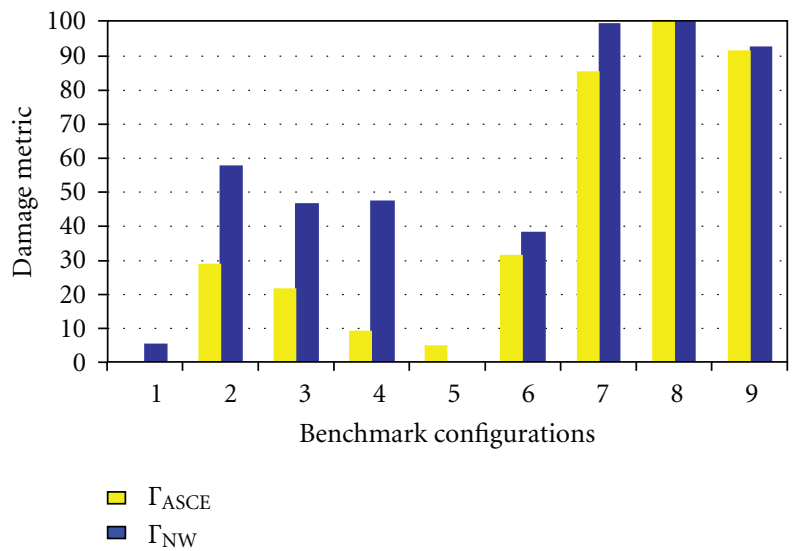

FIGURE 11: Neural-wavelet damage metric $\left(\Gamma_{\mathrm{NW}}\right)$ and descriptive damage metric $\left(\Gamma_{\mathrm{ASCE}}\right)$ for all ASCE benchmark structure configurations reported in Phase II-E (Configuration 5 is excluded).

more successful than the gradient descent method in realizing the optimal solution. The derivative-based technique was caught in local minima and the optimization process occasionally did not converge.

A comparison between the three optimal neural network architectures with one hidden layer denoted NN1 $\left(n_{1}=7\right.$ neurons), two hidden layers denoted NN2 $\left(n_{1}=1\right.$ and $\left.n_{2}=5\right)$ and three hidden layers denoted NN3 $\left(n_{1}=\right.$ 2 and $n_{2}=7$ and $n_{3}=10$ ) showed that the neural network NN3 with three hidden layers to have the highest classifier $(\rho)$. This comparison is shown in Figure 10. The comparison confirms that the optimal ANN architecture have the best ability to classify damage in the benchmark structure. The optimal ANN architecture shown in Figure 8 was used to identify damage in eight testing configurations of the ASCE benchmark structure (excluding Configuration 5 ) by computing the probability of damage (see (6)) in each configuration. This includes identifying damage in 
Configurations 2, 3, 4 and 9 that were not used in training the classifier.

Figure 11 shows the neural-wavelet damage metric $\Gamma_{\mathrm{NW}}$ of eight configurations as identified using the optimal damage classifier. The mean value and the standard deviation for each of the eight configurations was calculated as the mean of 14 time windows, each of them is eight seconds long. Figure 11 also shows the severity of damage metric $\Gamma_{\text {ASCE }}$ based on the damage quantification suggested in this paper (see (1)). Configurations 7, 8, and 9 that were classified as "fully damaged" (Table 2 ) show probabilities of damage ranging between 90 and 100 percent. The "partially damaged" Configurations 2, 3, 4, and 6 have a damage metric $\Gamma_{\mathrm{NW}}$ ranging between 40 and 60 percent and a descriptive damage metric $\Gamma_{\mathrm{ASCE}}$ between 9 and 31. The "healthy" Configuration 1 showed a damage metric $\Gamma_{\mathrm{NW}}$ that is lower than $5 \%$ and a descriptive damage metric $\Gamma_{\mathrm{ASCE}}$ value of zero. It is therefore obvious that a good agreement exists between the descriptive damage metric $\Gamma_{\mathrm{ASCE}}$ and the neural-wavelet damage metric $\Gamma_{\mathrm{NW}}$.

The above results provide a damage detection method that can detect damage in eight damage configurations of Phase II-E in the ASCE benchmark structure; the proposed damage metric can also be used to classify/quantify damage severity in the benchmark structure with reasonable accuracy. This is attributed to the fact that the damage feature was optimized for classification of damage. The optimization process correctly categorized the "partially damaged" benchmark configurations resulting in probabilities of damage that range from between 40 and 60 percent. The "fully damaged" configurations were also correctly classified for having probabilities of damage ranging between 90 and 100 percent. The optimal neural-wavelet method has proven capable of detecting damage occurrence and showed good sensitivity in quantifying damage severity for low and high damage configurations. However, the model showed less accuracy in quantifying partially damaged cases which might be enhanced if other configurations of partially damage data were used in establishing the classifier.

Finally, the proposed neural-wavelet framework can be applied to damage detection and classification of real world structures. This requires developing a finite element model to simulate the structure dynamic behavior and validate this model using historical data observed from the structure. Damage can then be introduced to the finite element model with different levels of damage severity and at different locations. This simulated data can be used to optimize the ANN and establish the neural-wavelet module. The developed neural-wavelet module can then be used to detect and classify damage in the real structure.

We need to emphasize, however, that the overall damage metric is not unique but provides an indicator to damage severity. It is important to realize that damage is a nonmeasurable quantity and damage metrics cannot be directly compared. This damage quantification inaccuracy is an intrinsic characteristic of damage related to the definition of damage as suggested by many researchers [65, 69-71]. Therefore, the use of absolute numbers for quantifying damage severity and the use of accuracy measures to validate damage quantification can lead to erroneous conclusions. Validation shall be limited to testing the metric ability to indicate the category of damage such as "low, moderate and high". The wide range of probabilities describing the damage level in each configuration is attributed to considering uncertainty in quantifying damage. It is obvious that damage quantification is a challenging problem that lends itself to probabilistic, fuzzy or imprecise quantification.

\section{Conclusion}

We demonstrated that it is possible to establish a damage pattern recognition method by designing a damage classifier that integrates ANN and WMRA. An optimization technique using derivative free optimization (genetic algorithm) was used to identify the optimal ANN architecture. A neural network, including three hidden layers with 2, 7, and 10 neurons in the first, second and third hidden layers respectively, was capable of successfully detecting and quantifying damage in the ASCE benchmark structure with a reasonable sensitivity.

The neural-wavelet method aimed at establishing the underlying relationships between the structural dynamic responses (acceleration signals) at the different locations of the structure during healthy performance then recognized changes in such relationships as damage advanced in the structure. While the use of ANN to learn the underlying relation of structural dynamics proved successful, some drawbacks of ANN are related to their intolerance to uncertainty in training data. It is therefore suggested that other learning methods with higher tolerance to classification uncertainty such as neural-fuzzy inference systems, adaptive fuzzy learning from examples [72] or support vector machines might be examined as an alternative to neural networks in realizing structural dynamic relationships.

\section{Acknowledgments}

The author greatly appreciates the financial support by Defense Threat Reduction Agency (DTRA). Special thanks to research assistants: Scott Horton, Molly McCuskey and Erdogan Altunok for their efforts in the SHM research projects with the author.

\section{References}

[1] F. K. Chang, Ed., Structural Health Monitoring: Current Status and Perspectives, Proceedings of the International Workshop on Structural Health Monitoring, Stanford, Calif, USA, 1997.

[2] D. L. Balageas, Ed., Structural Health Monitoring 2004: Proceedings of the 1st International European Workshop on Structural Health Monitoring, Paris, France, 2002.

[3] C. Boller and W. J. Staszewski, Eds., Structural Health Monitoring 2004: Proceedings of the 2nd International European Workshop on Structural Health Monitoring, University of Sheffield, Munich, Germany, 2004.

[4] A. Güemes, Ed., Structural Health Monitoring 2006: Proceedings of the 2nd International European Workshop on Structural Health Monitoring, Granada, Spain, 2006. 
[5] F. K. Chang, Ed., Structural Health Monitoring 2007: Quantification, Validation, and Implementation: Proceedings of the 6th International Workshop on Structural Health Monitoring, Stanford, Calif, USA, 2007.

[6] Los Alamos National Laboratory, "A review of structural health monitoring literature: 1996-2001," LA-13976-MS, 2004.

[7] E. A. Johnson, H. F. Lam, L. S. Katafygiotis, and J. L. Beck, "A benchmark problem for structural health monitoring and damage detection," in Proceedings of the 14th Engineering Mechanics Conference ASCE, Austin, Tex, USA, 2000.

[8] E. A. Johnson, H. F. Lam, L. S. Katafygiotis, and J. L. Beck, "Phase I IASC-ASCE structural health monitoring benchmark problem using simulated data," Journal of Engineering Mechanics, vol. 130, no. 1, pp. 3-15, 2004.

[9] S. J. Dyke, D. Bernal, J. L. Beck, and C. Ventura, "An experimental benchmark problem in structural health monitoring," in Proceedings of the 3rd International Workshop on Structural Health Monitoring, Stanford, Calif, USA, 2001.

[10] S. J. Dyke, J. M. Caicedo, and E. A. Johnson, "Monitoring of a benchmark structure for damage identification," in Proceedings of the ASCE Engineering Mechanics Conference, Austin, Tex, USA, 2000.

[11] A. Hera, Z. Hou, and M. Noori, "Wavelet-based approach for ASCE structural health monitoring benchmark studies," in Proceedings of 3rd International Workshop on Structural Health Monitoring, Stanford, Calif, USA, 2001.

[12] N. Y. Yang, S. Lin, and S. Pan, "Damage detection of a health monitoring benchmark building using Hilbert-Huang spectral analysis," in Proceedings of International Conference on Advances in Building Technology, pp. 1017-1024, Hong Kong, December 2002.

[13] A. Hera and Z. Hou, "Application of wavelet approach for ASCE structural health monitoring benchmark studies," Journal of Engineering Mechanics, vol. 130, no. 1, pp. 96-104, 2004.

[14] Z. Sun and C. C. Chang, "Structural degradation monitoring using covariance-driven wavelet packet signature," Structural Health Monitoring, vol. 2, no. 4, pp. 309-325, 2003.

[15] H. F. Lam, L. S. Katafygiotis, and N. C. Mickleborough, "Application of a statistical model updating approach on phase I of the IASC-ASCE structural health monitoring benchmark study," Journal of Engineering Mechanics, vol. 130, no. 1, pp. 34-48, 2004.

[16] K.-V. Yuen, S. K. Au, and J. L. Beck, "Two-stage structural health monitoring approach for phase I benchmark studies," Journal of Engineering Mechanics, vol. 130, no. 1, pp. 16-33, 2004.

[17] H. Luş, R. Betti, J. Yu, and M. De Angelis, "Investigation of a system identification methodology in the context of the ASCE benchmark problem," Journal of Engineering Mechanics, vol. 130, no. 1, pp. 71-84, 2004.

[18] J. M. Caicedo, S. J. Dyke, and E. A. Johnson, "Natural excitation technique and eigensystem realization algorithm for phase I of the IASC-ASCE benchmark problem: simulated data," Journal of Engineering Mechanics, vol. 130, no. 1, pp. 4960, 2004.

[19] D. Bernal and B. Gunes, "Flexibility based approach for damage characterization: benchmark application," Journal of Engineering Mechanics, vol. 130, no. 1, pp. 61-70, 2004.

[20] S. Lin, J. N. Yang, and L. Zhou, "Damage identification of a benchmark building for structural health monitoring," Smart Materials and Structures, vol. 14, no. 3, pp. S162-S169, 2005.
[21] J. G. Chase, V. Begoc, and L. R. Barroso, "Efficient structural health monitoring for a benchmark structure using adaptive RLS filters," Computers \& Structures, vol. 83, no. 8-9, pp. 639647, 2005.

[22] J. R. Wu and Q. S. Li, "Structural parameter identification and damage detection for a steel structure using a two-stage finite element model updating method," Journal of Constructional Steel Research, vol. 62, no. 3, pp. 231-239, 2006.

[23] J. N. Yang and H. Huang, "Sequential non-linear leastsquare estimation for damage identification of structures with unknown inputs and unknown outputs," International Journal of Non-Linear Mechanics, vol. 42, no. 5, pp. 789-801, 2007.

[24] Y. Mizuno and Y. Fujino, "Data archiving and processing method using wavelet decomposition for structural health monitoring," in Proceedings of the ASCE Conference on Computing in Civil Engineering, pp. 673-680, Pittsburgh, Pa, USA, 2007.

[25] W. Zhou, L. Mevel, and H. Li, "Stochastic subspace-based structural identification and damage detection-application to the ASCE benchmark structure," in Proceedings of the 6th International Workshop on Structural Health Monitoring, C. Fu-Kuo, Ed., pp. 645-652, Stanford, Calif, USA, 2007.

[26] Z. Hou and A. Hera, "Progress of phase II study of the ASCE health monitoring benchmark data using wavelet approach," in Proceedings of the 15th ASCE Engineering Mechanics Conference, Columbia University, New York, NY, USA, 2002.

[27] L. R. Barroso and R. Rodriguez, "Application of the damage index method to phase II of the analytical SHM benchmark problem," in Proceedings for the ASCE Engineering Mechanics Conference, New York, NY, USA, 2004.

[28] S. Casciati, "Statistical models comparision for damage detection using the ASCE benchmark," in Proceedings for the 2nd European Workshop on Structural Health Monitoring, C. Boller and W. J. Staszewski, Eds., pp. 695-701, University of Sheffield, Munich, Germany, 2004.

[29] A. Hera and Z. Hou, "Wavelet approach for damage detection using experimental data of ASCE benchmark study," in Proceedings of the ASCE Engineering Mechanics Conference, Seattle, Wash, USA, 2003.

[30] S. Dincal and A. Raich, "Structural damage detection using frequency response function," in Proceedings of the 5th International Workshop on Structural Health Monitoring, C. Fu-Kuo, Ed., Stanford, Calif, USA, 2005.

[31] K. K. Nair, A. S. Kiremidjian, and K. H. Law, "Time seriesbased damage detection and localization algorithm with application to the ASCE benchmark structure," Journal of Sound and Vibration, vol. 291, no. 1-2, pp. 349-368, 2006.

[32] J. Ching and J. L. Beck, "Two-stage Bayesian structural health monitoring approach for phase II ASCE benchmark studies," in Proceedings of the IMAC-XXI Conference, Orlando, Fla, USA, 2003.

[33] J. Ching and J. L. Beck, "Bayesian analysis of the phase II IASCASCE structural health monitoring experimetnal benchmark data," Journal of Engineering Mechanics, vol. 130, no. 10, pp. 1233-1244, 2004.

[34] D. Giraldo, J. M. Caicedo, and S. J. Dyke, "Experimental phase of the SHM benchmark studies. Damage detection using NeXT and ERA," in Proceedings of the 16th ASCE Engineering Mechanics Conference, Seattle, Wash, USA, 2003.

[35] J. P. Lynch, "Damage characterization of the IASC-ASCE structural health monitoring benchmark structure by transfer function pole Migration," in Proceedings of the ASCE Structures Congress and the Forensic Engineering Symposium, pp. 845854, New York, NY, USA, April 2005. 
[36] J. Liu, X. Wang, S. Yuan, and G. Li, "On Hilbert-Huang transform approach for structural health monitoring," Journal of Intelligent Material Systems and Structures, vol. 17, no. 8-9, pp. 721-728, 2006.

[37] M. M. McCuskey, M. M. Reda Taha, S. R. Horton, and T. J. Baca, "Identifying damage in the ASCE benchmark structure using a neural-wavelet module," in Proceedings of the 3rd European Workshop on Structural Health Monitoring, A. Güemes, Ed., pp. 421-429, Granada, Spain, 2006.

[38] M. C. McCuskey, Structural damage classification using optimization of a neural-wavelet module and possibility fusion, M.S. thesis, Department of Civil Engineering, University of New Mexico, Albuquerque, NM, USA, 2007.

[39] E. P. Carden and J. M. W. Brownjohn, "ARMA modelled timeseries classification for structural health monitoring of civil infrastructure," Mechanical Systems and Signal Processing, vol. 22, no. 2, pp. 295-314, 2008.

[40] M. B. Bischop, Neural Networks for Pattern Recognition, Oxford University Press, New York, NY, USA, 1996.

[41] P. Tsou and M. H. H. Shen, "Structural damage detection and identification using neural networks," AIAA journal, vol. 32, no. 1, pp. 176-183, 1994.

[42] R. S. Sexton, R. E. Dorsey, and J. D. Johnson, “Optimization of neural networks: a comparative analysis of the genetic algorithm and simulated annealing," European Journal of Operational Research, vol. 114, no. 3, pp. 589-601, 1999.

[43] M. F. Elkordy, K. C. Chang, and G. C. Lee, "A structural damage neural network monitoring system," Microcomputers in Civil Engineering, vol. 9, no. 2, pp. 83-96, 1994.

[44] E. Douka, S. Loutridis, and A. Trochidis, "Crack identification in beams using wavelet analysis," International Journal of Solids and Structures, vol. 40, no. 13-14, pp. 3557-3569, 2003.

[45] M. M. Reda Taha, A. Noureldin, J. L. Lucero, and T. J. Baca, "Wavelet transform for structural health monitoring: a compendium of uses and features," Structural Health Monitoring, vol. 5, no. 3, pp. 267-295, 2006.

[46] Z. Su and L. Ye, "An intelligent signal processing and pattern recognition technique for defect identification using an active sensor network," Smart Materials and Structures, vol. 13, no. 4, pp. 957-969, 2004.

[47] H.-F. Lam, K.-V. Yuen, and J. L. Beck, "Structural health monitoring via measured ritz vectors utilizing artificial neural networks," Computer-Aided Civil and Infrastructure Engineering, vol. 21, no. 4, pp. 232-241, 2006.

[48] K.-V. Yuen and H.-F. Lam, "On the complexity of artificial neural networks for smart structures monitoring," Engineering Structures, vol. 28, no. 7, pp. 977-984, 2006.

[49] R. O. Duda, P. E. Hart, and D. G. Stork, Pattern Classification, Wiley Interscience, New York, NY, USA, 2001.

[50] L. H. Yam, Y. J. Yan, and J. S. Jiang, "Vibration-based damage detection for composite structures using wavelet transform and neural network identification," Composite Structures, vol. 60, no. 4, pp. 403-412, 2003.

[51] H. Kim and H. Melhem, "Damage detection of structures by wavelet analysis," Engineering Structures, vol. 26, no. 3, pp. 347-362, 2004.

[52] Y.-S. Diao, H.-J. Li, and Y. Wang, "A two-step structural damage detection approach based on wavelet packet analysis and neural network," in International Conference on Machine Learning and Cybernetics, pp. 3128-3133, August 2006.

[53] X. Jiang and H. Adeli, "Pseudospectra, MUSIC, and dynamic wavelet neural network for damage detection of high-rise buildings," International Journal for Numerical Methods in Engineering, vol. 71, no. 5, pp. 606-629, 2007.
[54] X. Jiang and S. Mahadevan, "Bayesian probabilistic inference for nonparametric damage detection of structures," Journal of Engineering Mechanics, vol. 134, no. 10, pp. 820-831, 2008.

[55] X. Jiang, S. Mahadevan, and R. Guratzsch, "Bayesian wavelet methodology for damage detection of thermal protection system panels," AIAA Journal, vol. 47, no. 4, pp. 942-952, 2009.

[56] H. Sohn, D. W. Allen, K. Worden, and C. R. Farrar, "Structural damage classification using extreme value statistics," Journal of Dynamic Systems, Measurement and Control, vol. 127, no. 1, pp. 125-132, 2005.

[57] E. Altunok, M. M. Reda Taha, and T. J. Ross, "Possibilistic approach for damage detection in structural health monitoring," Journal of Structural Engineering, vol. 133, no. 9, pp. 1247-1256, 2007.

[58] ASCE Benchmark Group, http://wusceel.cive.wustl.edu/asce. shm/benchmarks.htm.

[59] E. Altunok, M. M. Reda Taha, D. S. Epp, R. L. Mayes, and T. J. Baca, "Damage pattern recognition for structural health monitoring using fuzzy similarity prescription," ComputerAided Civil and Infrastructure Engineering, vol. 21, no. 8, pp. 549-560, 2006.

[60] M. M. Reda Taha, A. Noureldin, A. Osman, and N. El-Sheimy, "Introduction to the use of wavelet multiresolution analysis for intelligent structural health monitoring," Canadian Journal of Civil Engineering, vol. 31, no. 5, pp. 719-731, 2004.

[61] S. G. Mallat, A Wavelet Tour of Signal Processing, New York Academic, New York, NY, USA, 1999.

[62] M. Haase and J. Widjajakusuma, "Damage identification based on ridges and maxima lines of the wavelet transform," International Journal of Engineering Science, vol. 41, no. 13-14, pp. 1423-1443, 2003.

[63] A. V. Ovanesova and L. E. Suárez, "Applications of wavelet transforms to damage detection in frame structures," Engineering Structures, vol. 26, no. 1, pp. 39-49, 2004.

[64] S. Horton, M. M. Reda Taha, and T. J. Baca, "A neural-wavelet damage detection module for structural health monitoring," in Proceedings of 5th International Workshop on Structural Health Monitoring, Stanford, Calif, USA, 2005.

[65] C. R. Farrar and D. A. Jauregui, "Comparative study of damage identification algorithms applied to a bridge: II. Numerical study," Smart Materials and Structures, vol. 7, no. 5, pp. 720731, 1998.

[66] J. Li, Y. Zhang, and S. Zhu, "A wavelet-based structural damage assessment approach with progressively downloaded sensor data," Smart Materials and Structures, vol. 17, no. 1, Article ID 015020, 2008.

[67] G. Vanderplatts, Numerical Optimization Techniques for Engineering Design with Applications, McGraw-Hill, New York, NY, USA, 1984.

[68] R. L. Haupt and S. E. Haupt, Practical Genetic Algorithms, John Wiley \& Sons, New York, NY USA, 1998.

[69] S. Toussi and J. T. P. Yao, "Assessment of structural damage using the theory of evidence," Structural Safety, vol. 1, no. 2, pp. 107-121, 1982.

[70] M. M. Reda Taha and J. Lucero, "Damage identification for structural health monitoring using fuzzy pattern recognition," Engineering Structures, vol. 27, no. 12, pp. 1774-1783, 2005.

[71] M. Chandrashekhar and R. Ganguli, "Uncertainty handling in structural damage detection using fuzzy logic and probabilistic simulation," Mechanical Systems and Signal Processing, vol. 23, no. 2, pp. 384-404, 2009.

[72] T. J. Ross, Fuzzy Logic with Engineering Applications, John Wiley \& Sons, London, UK, 2004. 

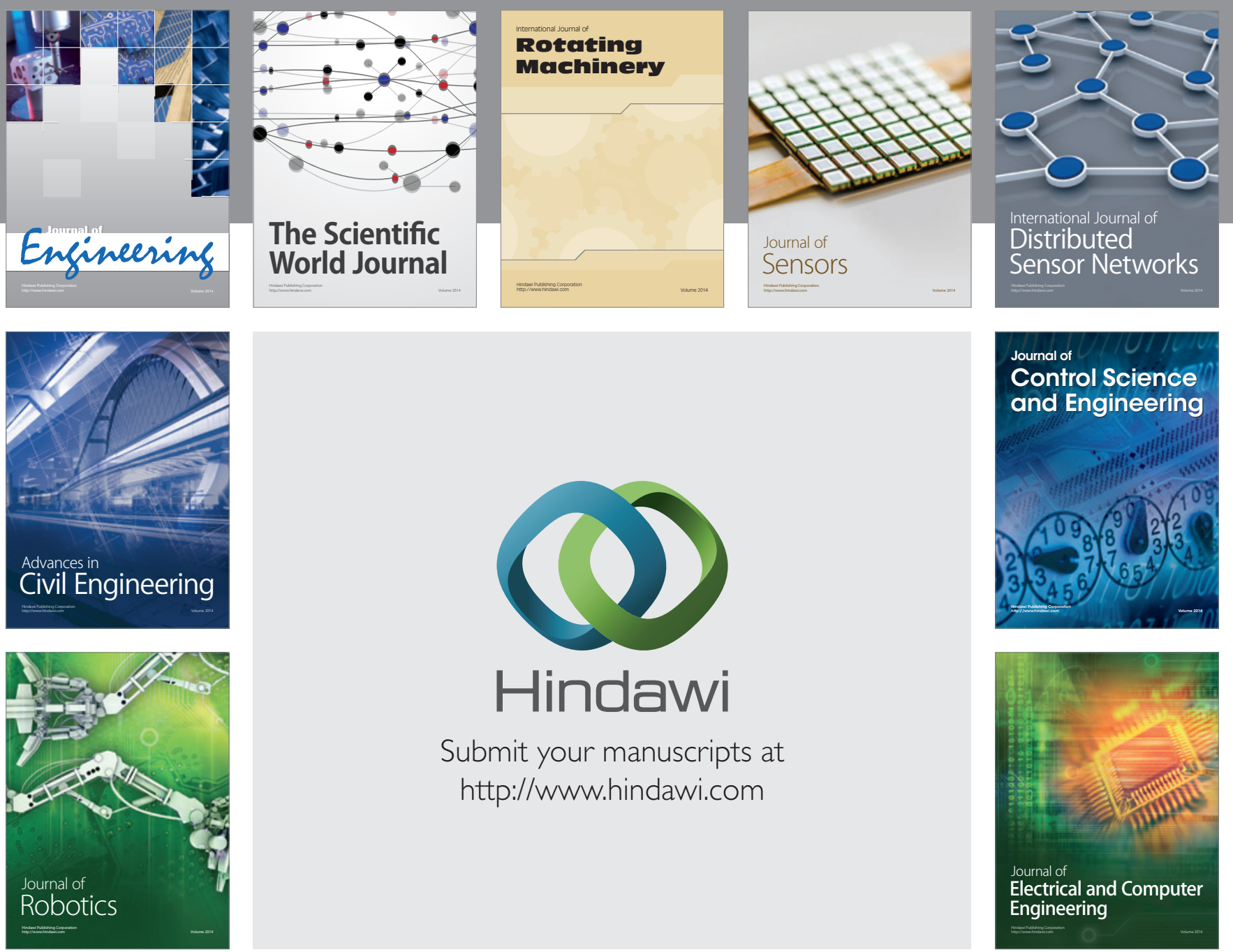

Submit your manuscripts at

http://www.hindawi.com
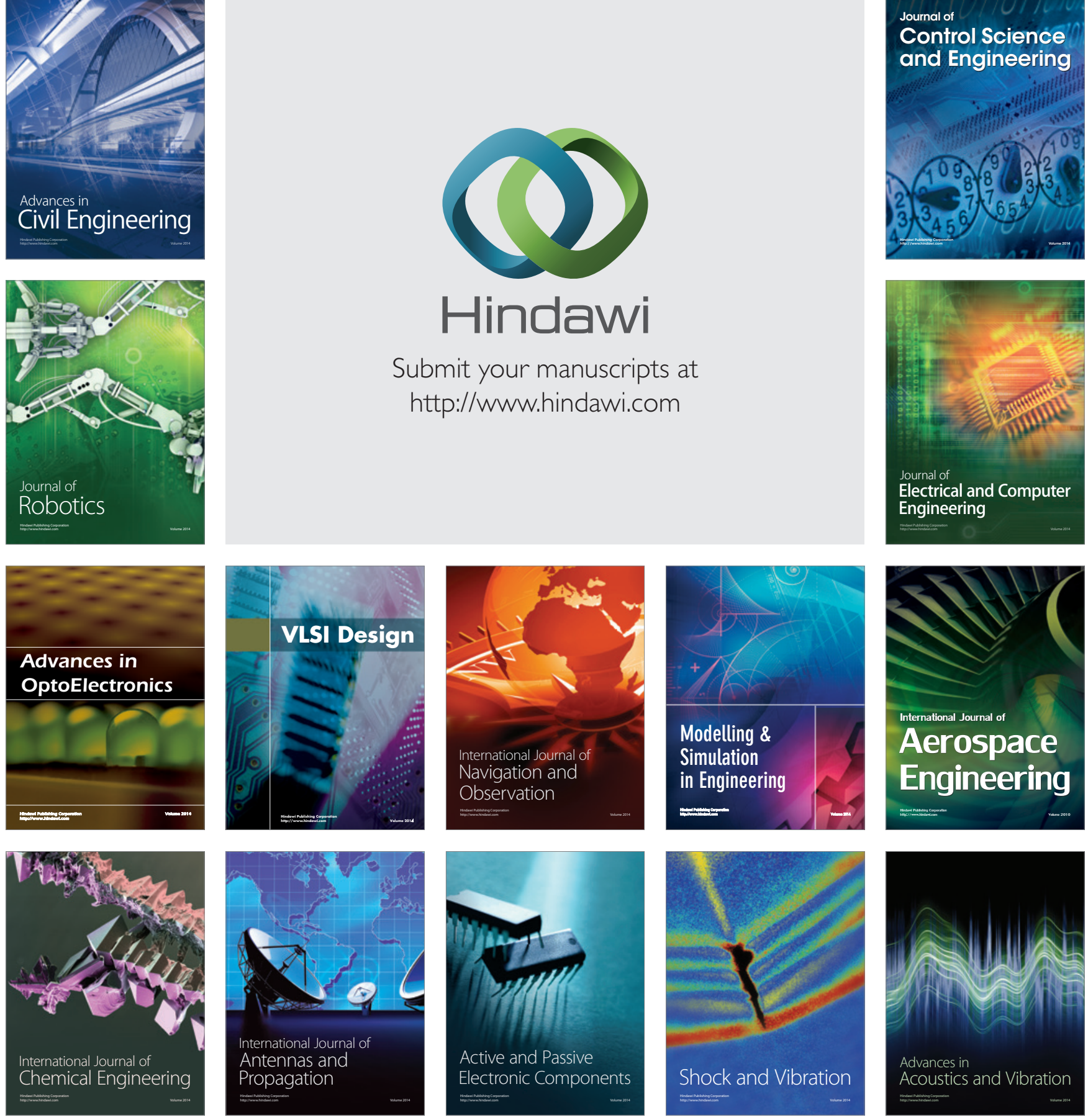